\title{
Identification of unrecognizable corpses: A novel semi-automated approach for forensic facial reconstruction in Sri Lanka
}

Vidanapathirana $\mathrm{M}^{1}$, Rajapakse R. $\mathrm{N}^{2}$, Madugalla A.K $\mathrm{K}^{2}$, Amarasinghe P.I. $\mathrm{U}^{2}$, Padmathilake V. $H^{2}$, Dharmaratne A. $T^{2}$,Sandaruwan K. $D^{2}$

${ }^{1}$ Department of Forensic Medicine, Faculty of Medical Sciences, University of Sri Jayawardhanapura, Sri Lanka, ${ }^{2}$ University of Colombo School of Computing, Colombo, Sri Lanka

*Corresponding author: Tel: 0094-718244641. E-mail address: mudithavidana@yahoo.co.uk

MLJSL. Vol 1. No 2. Aug. pp $10-22$

\begin{abstract}
Introduction: Identification of unrecognizable corpses is of high importance and when all other identification mechanisms fail, the process of forensic facial reconstruction has to be adopted.

Objective: This process is still at its infancy in Sri Lanka and is yet to utilize the advanced technologies of other countries. Hence introducing a more efficient, semi-automated 3D Computer graphics based technique for the local forensic officials is the aim of this study.
\end{abstract}

Method: A 3D model of the skull is captured and digitally sculpting facial muscles on the model with the aid of forensic facial markers. Different facial components were added to the completed face model in order to improve the identification process. Separate analyses were also conducted for both facial tissue thickness and facial component variations in Sri Lankans to achieve an improved result. Female cases of the age category 20-30/ medium weight were used in this study.

Results: The analysis of the facial tissue thickness confirmed that tissue thickness data of other countries cannot be adopted for facial reconstruction in the local context. Sri Lankans have a different facial soft tissue thickness mainly in the following areas; Gonion, Sub M2, Supra M2 and the area beneath the chin. The facial feature analysis discovered the mean values of the nasal and eye indexes which were then modeled in to the final output. The outputs were thoroughly evaluated using a number of techniques.

Conclusion: By adopting the suggested novel application based on the evaluation results and the cost analysis, establishing the first unit for facial reconstruction in Sri Lanka would be highly recommended to uphold the rights of deceased and relatives concerned.

Keywords: Forensic facial reconstruction, muscle-anatomy, facial components, Sri Lankan, tissue thickness, digital sculpting, 


\section{Introduction}

Identification of unrecognizable corpses is of high importance. The right to be buried in accordance with one's religion and traditions in a grave that bears one's own name is a basic human right that each person possesses. That right is closely similar to the rights of the relatives of a missing person to know the status of their loved ones.

Manmade or natural disasters such as terrorist bombings, mob violence and Tsunami, etc., result in innumerable unidentified dead bodies. Murder victims plunged deep underground and surfaced years later would also be unidentifiable dead bodies. In such situations, when all other identification methods fail, investigations are directed towards facial reconstruction stage. Once a face is reconstructed it is circulated via digital and print media with the hope of someone being able to identify it. Upon such identification, the stated identification is verified using mechanisms such as DNA or dental records analyses.

Yet it has to be borne in mind that this stage is also known as the facial approximation, because the end result of this process, is a face that would only hold some resemblance to the deceased, and will not be the exact face of the person.

Although forensic facial reconstruction is used in a very productive manner in the global context, our country is at it's infancy. Years back, Prof GSW de Saram and Mr. Webster from Faculty of Medicine, Colombo conducted superimposition and facial reconstruction projects using manual techniques, some cases were presented and challenged in a court of law. Hence introducing a more efficient multimedia based technique to the local forensic officials in order to improve the efficiency and the accuracy of the reconstruction process is the aim of this study. This research study introduces a novel semiautomated method for Forensic facial reconstruction.

Part of the findings of this research was published in Galle Medical Journal.

\section{Method}

Several human skulls along with the photographs of the deceased were collected from Medical Faculties of Sri Jayewardenepura and Karapitiya for evaluation purposes.

The process was carried out with a prerequisite stage and four main steps as follows.

Pre-requisites:

A. Forensic Analysis of the Skull of the deceased person,

B. Ascertainment of facial tissue thicknesses,

C. Facial component analysis

Step 01: Capturing the 3D model of the skull[1]

Step 02: Placement of Landmarks

Step 03: Digitally Sculpting the Face

Step 04: Adding Facial Components

Step 05: Evaluation of identification

Pre-requisite: A. Forensic analysis of the skull

An expert in Forensic Medicine, ascertained the following details from the skeletal remains. 
1. Age category (An assumption in a bracket of 5-10 years would suffice) E.g. 20-30

2. Sex

Those ascertained features assisted the subsequent reconstruction process.

\section{Pre-requisite: B. Ascertainment of facial} tissue thicknesses

Forensic cases where the deceased was of medium weight, 20-30 years old, females were chosen and so were the tissue thickness samples. 31 anatomic and physiognomic relevant points (tissue thickness landmarks) were identified on the skull (Figure 1). The number of landmarks was determined by the research team to suit the nature of the muscle sculpting method. Previous studies in this regard have been performed with differing numbers of landmarks. [2,3,4,5]

Samples of 30 were created from females of the age category 20-30. Tissue thickness depths for each of those anatomical landmarks were obtained by using MRI and CT images of the sample group (Figure 1). For this purpose, Head/ Sinus scans (without the patient identification details) obtained from medical institutions were used.

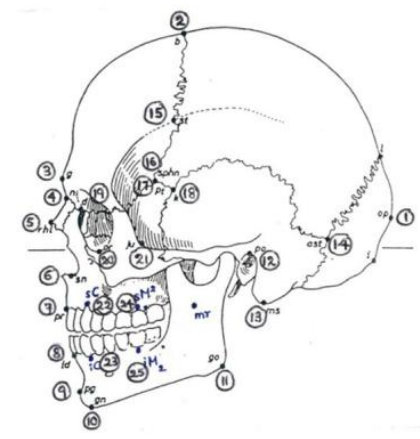

Figure 1: Lateral view of the skull showing landmarks from 1-31
Following the tissue thickness analysis, it was found that there is a considerable difference between the facial soft tissue thicknesses depths of Sri Lankans and other races at the areas of beneath the Chin, Gonion, Sub M2 (Below the second mandibular molar) and Supra M2 (Above the second maxillary molar) (Figure 2).

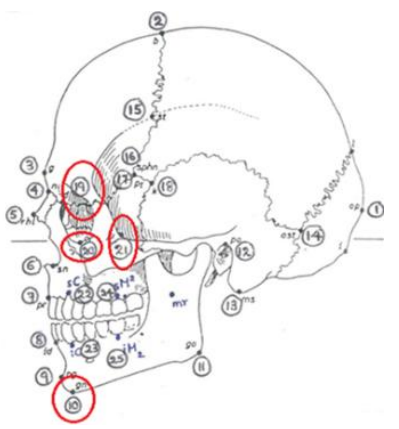

Figure 2: Areas of differing tissue thickness in Sri Lankans as compared with the data in other regions.

Therefore, applying tissue thickness values of other countries to a Sri Lankan skull model would result in inaccurate approximations.

\section{Prerequisite: C. Facial component analysis}

The mean values of nasal and eye indices were calculated for facial component analysis. Noses and eyes of a sample more than 400 volunteers of the age category 20 30 was photographed for this analysis. Facial components were divided under three categories; narrow, medium and broad.

NOSE ANALYSIS

The Nasal Index was calculated applying the following "Broca Agreement" formula using the photographs of the sample group.

$$
\text { Nasal Index (N.I) }=\frac{\text { Nose width }}{\text { Nose height }} \times 100
$$


EYE ANALYSIS

"Halder Method" was used to measure variations in the eyes.

$$
\text { Eye Index }(E . I)=\frac{\text { Eye width }}{\text { Eye height }} \times 100
$$

\section{Step 01: Capturing the 3D model of the skull}

A Vivid 910 3D scanner of Konica Minolta ${ }^{\mathrm{TM}}$ was used to capture the skull (Figure 3).

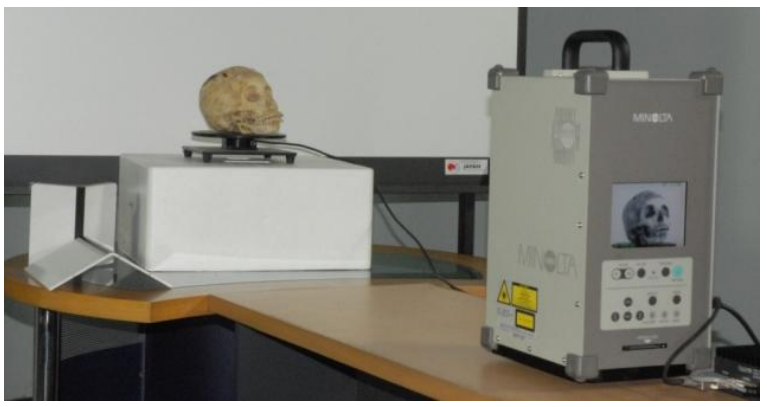

Figure 3: The set up of acquiring 3D model of the skull with the Vivid 910 3D Laser scanner

This scanner has already been used to generate models of human components in the Medical Field.[1]

Once the $3 D$ models were acquired using the 3D Scanner, it was observed that most of the test skulls had fractures and were damaged. This was not an unexpected dilemma since most of the human remains unearthed after years would be in a decayed stage.

However this status of the skulls rendered it hard to conduct an accurate reconstruction. Hence it was required to make certain rectifications to the $3 \mathrm{D}$ models of the skulls. A 3D editing software application was utilized in this stage to successfully rectify the acquired 3D models. (Figure 4 [a \& b]).
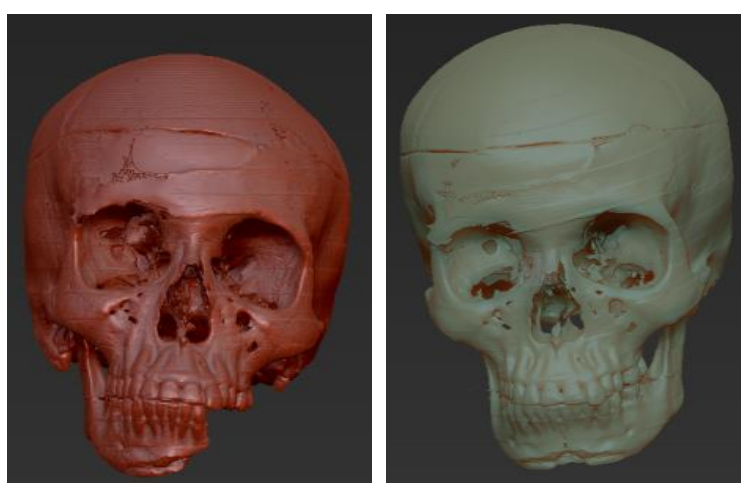

Figure 4: (a) Test skull (Case 01) with half of the jaw missing (b) After applying the symmetry modifier in a 3D editing software application

\section{Step 02: Placement of Landmarks}

For this, tissue thickness data obtained from a samples of people who were similar to the deceased in terms of age and sex were used (figure 5) by using 3DX Max software application.
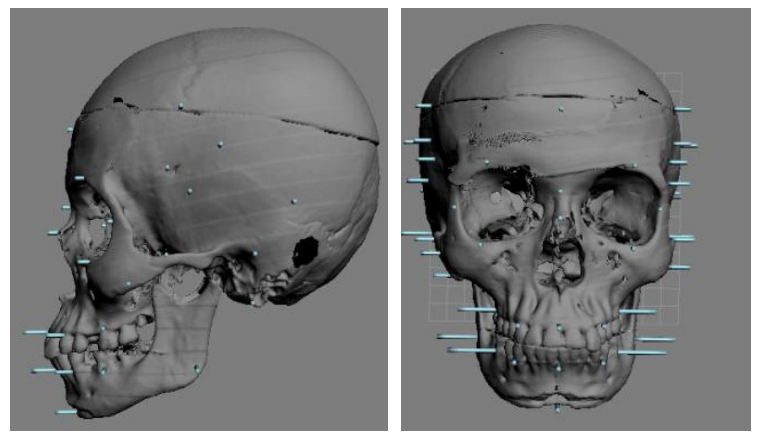

Figure 5: The skull model with the tissue thickness landmarks (Case 01)

\section{Step 03: Digitally Sculpting the Face}

By using those depth values, the height of the tissue thickness landmarks placed on the model were then adjusted. In contrast to the other mechanisms adopted in facial reconstruction in other studies, this study used a novel facial muscle sculpting based approach for reconstruction. 
Several techniques were tried out and it was decided to use the $\mathrm{ZBrush}^{\mathrm{TM}}$ software for the muscle reconstruction since it is digital sculpting software. Each facial muscle was carefully constructed and the thicknesses of each of these muscles were determined by the landmarks (figure 6 ).
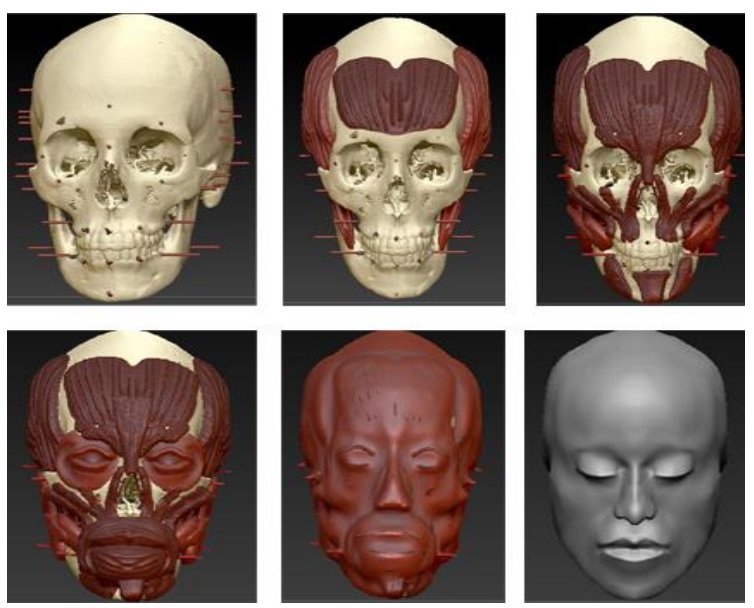

Figure 6: The different stages of the reconstruction procedure (Case 02female/20-30)

The sculpting of the basic muscles was followed by the construction of more complex facial components such as eyes and nose along with applying skin on top of all the muscles (figure 7).

\section{Step 04: Adding Facial Components}

A realistic appearance for the reconstructed face was obtained by adding modeled facial components such as eyes and noses appropriately.

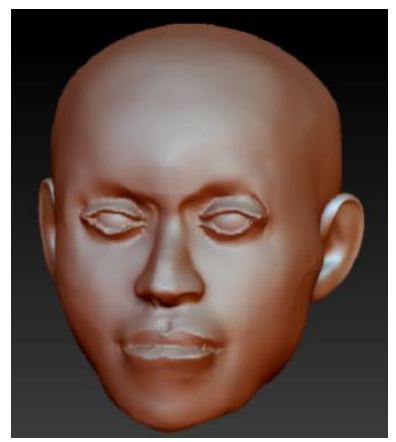

Figure 7: The Intermediate model created using the muscular structure (Case 01)

As facial feature data from other regions would not be appropriate, a separate facial feature analysis was conducted on Sri Lankans. Using the mean reference values of Nasal and Eye Indices, facial components (different sizes and shapes) (Refer Table II, III) were added to the 3D model to improve the possibility of identification (figure 8).

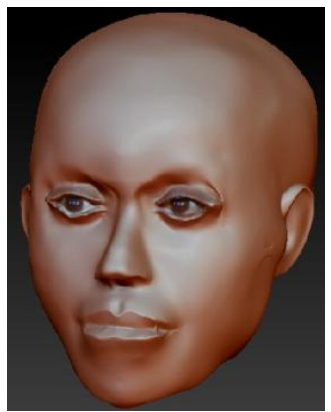

Figure 8: The Final model of the process (Case 01)

\section{Step 05: evaluation of final appearance}

The method adopted to reconstruct the face resulted in a 3D face model that had a fair resemblance to the deceased person's actual photograph. This was evaluated using both non-technical and technical evaluation techniques to ensure the accuracy.

"Face pool comparison", was the non technical evaluation used for digital photos. The reconstructed face and four other photographs with actual photograph of the deceased were emailed among more than 100 university students (figure 9) (http://facereconsurvey.appspot.com/).
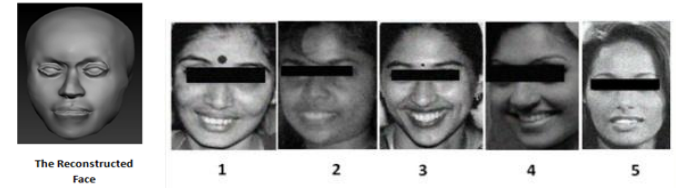
Figure 9: Face pool comparison for digital photos evaluation. The actual image of the deceased is the No.02.

The non-technical evaluation technique used for CT photos was the "Resemblance Rating". CT photo with the reconstructed face images were emailed among university students

(figure

10)

(http://facereconsurvey2.appspot.com/ ).

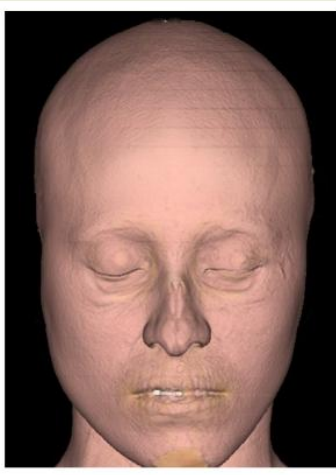

a.) Actual Image

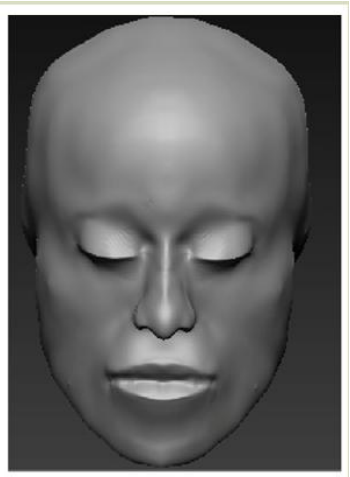

b.)Reconstructed 3D Model
Figure 10: "Resemblance rating technique" for CT photo evaluation.

Under the technical evaluation Photo Superimposition and Photogrammetry Analysis were adopted.

P. Vanezis (2000) in his 3-D computer graphics facial reconstruction too used superimposition (figure 11).

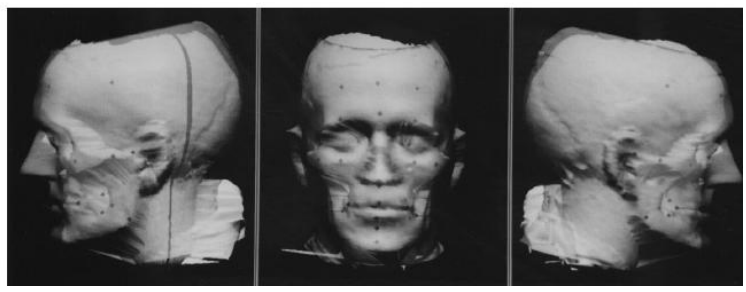

Figure 11: 3-D computer graphics facial reconstruction by $P$. Vanezis in 2000

In our study, a 3D editing software was used to find the orientation and to superimpose. In the superimposition technique which is a widely accepted way of evaluating outputs of this sort [2], the image of the model was placed on top of the actual photo to analyze whether the contours match. This allowed to verify the soft tissue alignment and to see if there were any obvious errors (figure 12).
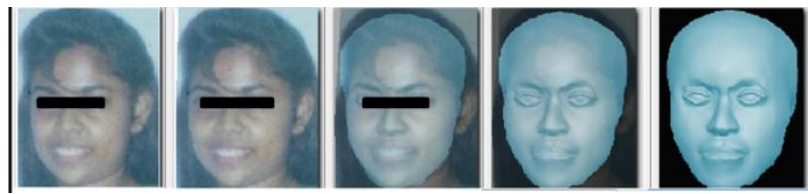

Figure 12: Superimposition views of the model with the actual photograph of the deceased.

The final superimposition figure was evaluated by Free-morphing Superimposition evaluation technique.

In the "Photogrammetry analysis", the $2^{\text {nd }}$ technical evaluation method, the distance between defined facial components were measured and compared between the model and the actual photograph (figure 13). The Photogrammetry Analysis was done by using facial recognition software.

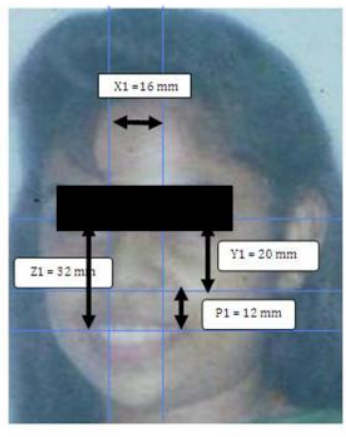

Actual Image (A1)

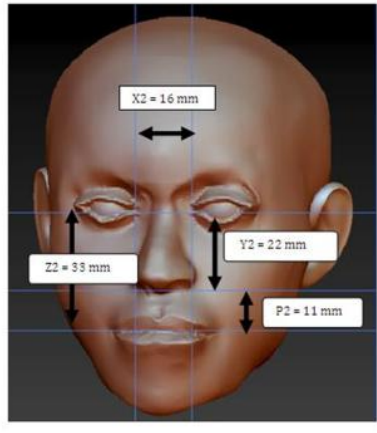

Reconstructed 3D Model (A2)
Figure 13: "Photogrammetry Analysis" for evaluation. 


\section{Results}

The results of the process and evaluation techniques of the study are as follows.

\section{Result1: facial tissue thicknesses}

Table 1 shows the tissue thickness values that were gathered using MRI and CT scans of medium weight female of 20-30 years (table I).

TABLE I

Mean Facial Tissue Depth Data for SRi lankan FEMALE AGED 20-30

\begin{tabular}{|l|l|}
\hline $\begin{array}{l}\text { Point Number/Name of } \\
\text { the Landmark }\end{array}$ & $\begin{array}{l}\text { Mean Values } \\
(\mathrm{mm})\end{array}$ \\
\hline 1.Opisthocranion & 5.87 \\
\hline 2. Bregma & 5.49 \\
\hline 3.Glabella & 3.25 \\
\hline 4.Nasion & 5.32 \\
\hline 5.End of Nasal bone & 3.22 \\
\hline 6.Mid-philtrum & 10.58 \\
\hline 7.Upper lip margin & 10.02 \\
\hline 8.Lower lip margin & 10.2 \\
\hline 9.Mental eminence & 9.41 \\
\hline 10.Beneath Chin & 17.08 \\
\hline 11.Gonion & 23.98 \\
\hline 12.Supra glenoid & 10.95 \\
\hline 13.Mastoidale & 8.75 \\
\hline 14.Asterion & 7.84 \\
\hline 15.Stephanion & 5.77 \\
\hline 16.Sphenion & 7.91 \\
\hline 17.Pterion & 10.23 \\
\hline 18.Krotaphion & 10.48 \\
\hline 19.Supra orbital & 5.5 \\
\hline 20.Infra orbital & 6.35 \\
\hline 21.Jugale & 9.66 \\
\hline 22.supra-Canine & 8.33 \\
\hline 23.infra-Canine & 19.87 \\
\hline 24.Supra M2 & 25.2 \\
\hline 25.Sub M2 & 23.4 \\
\hline 26.Euryon & 8.03 \\
\hline 27.Inferior malar & 12.8 \\
\hline & \\
\hline
\end{tabular}

\begin{tabular}{|l|l|}
\hline 28.ectocochion & 3.37 \\
\hline 29.Zygomatic arch & 9.33 \\
\hline 30.Maxillofrontale & 9.03 \\
\hline 31.Occlusal line & 20.23 \\
\hline
\end{tabular}

\section{Result 2: Facial component analysis}

For this analysis a sample of 426 (290 males and 136 females) of the age category 20-30 was used, divided under three categories in facial components and were devised.

NOSE ANALYSIS

Nasal index of 20-30 years females are shown below.

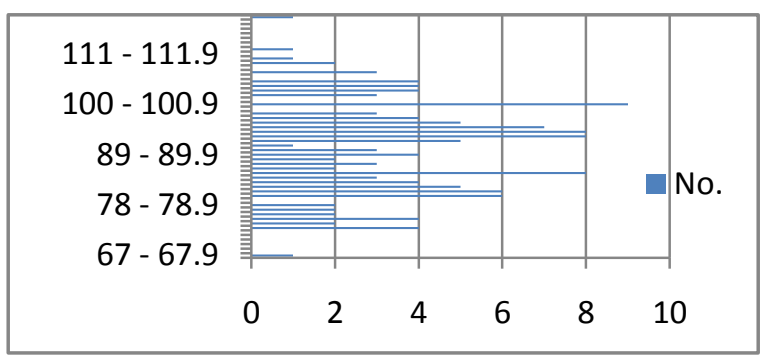

After which the indices were categorized as narrow, medium and broad and the mean $\mathrm{N}$.I values for each of the categories were calculated. (Table II)

TABLE II

The Mean Values of Nasal Index Data for SRI LANKAN FEMALES AGED 20-30

EYE ANALYSIS

\begin{tabular}{|l|l|l|}
\hline $\begin{array}{l}\text { Nose } \\
\text { Categories }\end{array}$ & $\begin{array}{l}\text { Mean N.I } \\
\text { (Male) }\end{array}$ & $\begin{array}{l}\text { Mean N.I } \\
\text { (Female) }\end{array}$ \\
\hline Narrow & 100 & 80 \\
\hline Medium & 103 & 100 \\
\hline Broad & 136 & 104 \\
\hline
\end{tabular}

Eye index of 20-30 years females were as follows. 


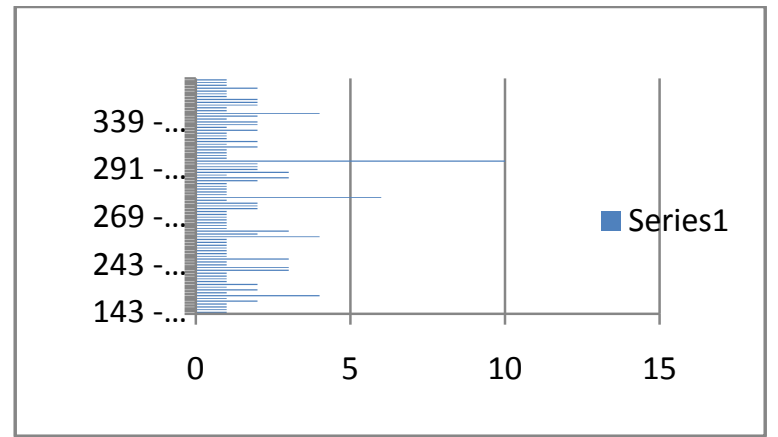

After which the indices were categorized as narrow, medium and broad and the mean E.I values for each of the categories were calculated. (Table III)

TABLE III

The Mean Values of Eye Index Data for SRI LANKAN FEMALES AGED 20-30

\begin{tabular}{|l|l|l|}
\hline $\begin{array}{l}\text { Eye } \\
\text { Categories }\end{array}$ & $\begin{array}{l}\text { Mean E.I } \\
\text { (Male) }\end{array}$ & $\begin{array}{l}\text { Mean E.I } \\
\text { (Female) }\end{array}$ \\
\hline Narrow & 300 & 257 \\
\hline Medium & 355 & 300 \\
\hline Broad & 733 & 400 \\
\hline
\end{tabular}

\section{Result 3: Evaluations}

In "Face Pool Comparison", the NonTechnical Evaluation method used for Digital photos, 117 responded. More than $50 \%$ of the respondents managed to successfully select the correct image (Figure 14: $2^{\text {nd }}$ person). Other evaluation surveys showed similar results.

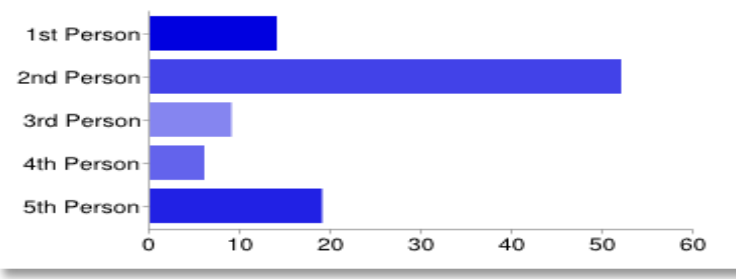

Figure 14: "Face Pool Comparison" Survey results of the case 01

In "Resemblance Rating", the Non-Technical Evaluation technique used for CT photos, 34 responded. Almost all responded that the reconstructed image was either similar or identifiable to the CT photos. Case 03 evaluation survey showed similar results (Figure 15).

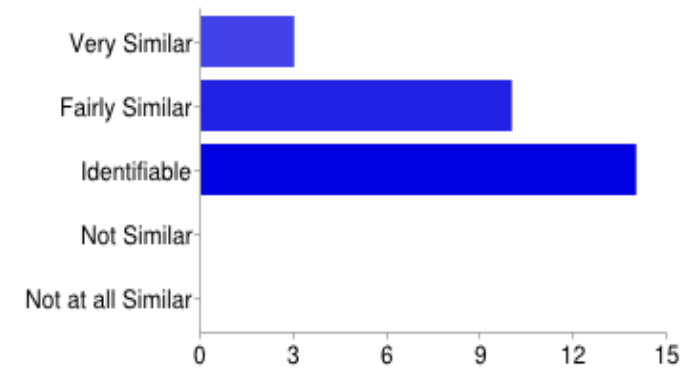

Figure 15: "Resemblance Rating" survey results of case 03

Photogrammetry Analysis, a Technical Evaluation technique, by using facial recognition software showed significant similarity in all 4 cases. eg. Figure 16 shows the Photogrammetry analysis for case 01 . 

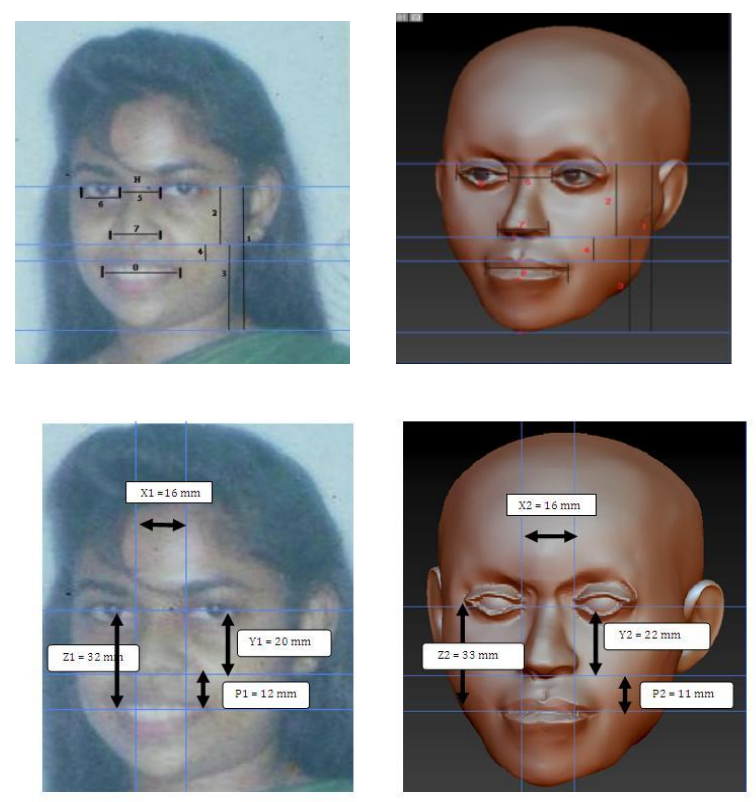

Actual Image (A1)

Reconstructed 3D Model (A2)

Figure 16: Photogrammetry Analysis for case 01.

Results of the "Superimposition", the $2^{\text {nd }}$ Technical Evaluation method also showed significant similarity in all 4 cases, so as in case 01 (Figure 12). Free-morphing software application was used to evaluate the superimposition technique and it showed perfect match in all 4 cases. Eg. in case 01 (figure 17).

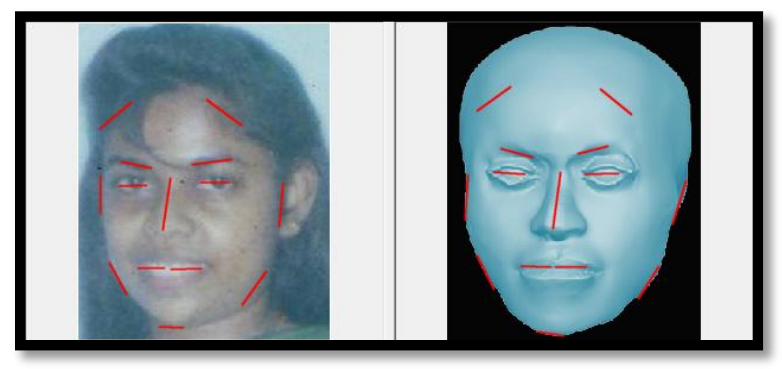

Figure 17: Free-morphing Superimposition evaluation technique

Both these technical evaluation methods resulted in depicting that the difference between the reconstructed face and the deceased persons photograph to be negligible.

\section{Discussion}

There is a standard procedure that is followed by forensic officials when they receive an unrecognizable corpse. First the bodies are allowed for recognition by facial features by the relatives or friends and usually, fresh bodies are identified by facial recognition. With putrefaction, facial recognition may not be possible. In failing that, the forensic officials would ascertain general and tentative identification of the dead bodies. In 'general identification' forensic officials will estimate values for age, sex, race and stature. For 'tentative identification', documents, ornaments, clothes, scars, tattoos, stigmata, habits, occupational stigmata, deformities, radiological findings are documented. These information will then be published in media and also compared with missing persons' data base. If a relative or friend comes forward, they are asked to submit antemortem data such as X-rays, medical records, dental records etc of their missing person. Those facts can be used to confirm the identification by using primary identifiers; Dental, Radiology, DNA and finger prints techniques.

What would happen if no relatives or friends came forward? Those unidentified remains would remain unidentified. Eg. if an isolated skull is found, relatives or friends may not be able to Identify same. In exhumations too, the skeletons may not be identified. In such instances, unidentified remains are buried after 14 days, following all faith ceremony.

Therefore, the ultimate solution for unidentified remains is facial reconstruction. The reconstructed face is published in media and is compared with missing persons data base. The relatives or 
friends may be able to identify the reconstructed face and we get the opportunity to obtain antemortem data of the missing person and then the identification can be confirmed by using primary identifiers. Finally, a funeral can be arranged according to the deceased's faith and disposed upholding their dignity.

Facial reconstruction was not implemented in Sri Lanka due to non availability of facial tissue thickness data and facial component (eyes, nose) data, and high cost of purchasable automated solutions. Manual method of facial reconstruction is time consuming, less productive and also expertise is lacking.

The goals of our research were to find a feasible, computer based, 3D, Semiautomated facial reconstruction solution to Sri Lanka.

This study encompassed reconstructions where the sources were taken from CT scans of living people to increase the number of reconstructions done. In these cases the skull and the actual photograph of the face was acquired using surface rendering DICOM images of CT scans (figure 18).

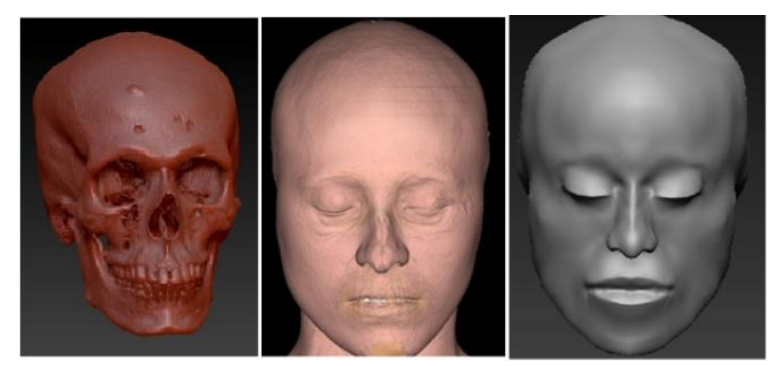

Figure 18: A CT scan case

Since 3D scanners are not commonly available, it was found that using CT images of the skull can also be used as a viable option to generate 3D models. The advantage of this method is that since the CT scanner possesses the capability to capture details precisely, the resulting model would be of high accuracy and detail. The DICOM output of the CT scanner was imported in to an image processing application dedicated to DICOM images [Osirix-viewer] and the 3D model was obtained.

The recommended semi-automated methodology of reconstructing based on facial muscles; especially in the field of computer based 3D reconstruction is a novel approach. The literature stated that the manual reconstruction method resulted in a natural looking output but lacked in efficiency and expertise. Further, we found that a fully automated technique would be more efficient compared to the manual technique. But the output due to the process being fully automated resulted in an unrealistic final model. Thus it was decided to use a semi-automated technique by combining the advantages of both manual and fully automated techniques. This methodology automates the steps of the reconstruction process where inefficiencies occurred in the manual process. E.g Creating a plasticine mould of the skull, sculpting muscles manually using modeling clay. Since the manual reconstruction is done mostly by modeling the facial muscles on a model of the skull, the authors were of the opinion that having a direct map between this technique and the computerized 3D reconstruction process would result in a more natural looking output. Thus muscles were sculpted manually in a digital environment which unlike in the fully automated process resulted in a more natural and realistic output. 
Since both evaluations gained positive results as well, reconstructing the face using the muscle-based anatomy approach was proven to be reliable and accurate to a sufficient extent. The following problems were addressed in this research (Table IV).

TABLE IV

SPECIFIC PROBLEMS ADDRESSED BY THIS RESEARCH

\begin{tabular}{ll}
\hline Problem & Solution \\
\hline $\begin{array}{l}\text { 1. Lack of Tissue } \\
\text { thickness data of } \\
\text { Sri Lankans }\end{array}$ & $\begin{array}{l}\text { Tissue Thickness } \\
\text { Analysis }\end{array}$ \\
$\begin{array}{l}\text { 2. Lack of facial } \\
\text { features differences } \\
\text { data on Sri Lankans }\end{array}$ & Facial Feature \\
$\begin{array}{l}\text { 3. Expert technical } \\
\text { knowledge on facial }\end{array}$ & 3D sculpting based \\
reconstruction & \\
process & \\
$\begin{array}{l}\text { 4. High Cost of } \\
\text { exiting solutions }\end{array}$ & 700 \$ software cost \\
5. Time Constraint & 4-5 Hours \\
\hline
\end{tabular}

After the cost analysis was done it was quite apparent that the solution presented was feasible especially in monetary terms. The cost of the software used, amounts to $\$ 700$ and that compared with the systems used in other countries (UK $\$ 75,000$ ) is a very low cost solution. The time consumed was also considerably less when compared with the manual technique. Furthermore, the expertise in 3D modeling would not be an issue either due to the reason that to a 3D modeling artist the muscle sculpting learning curve would be substantially short. Comprehensive manuals and documentation have been created by the research team (figure 19).

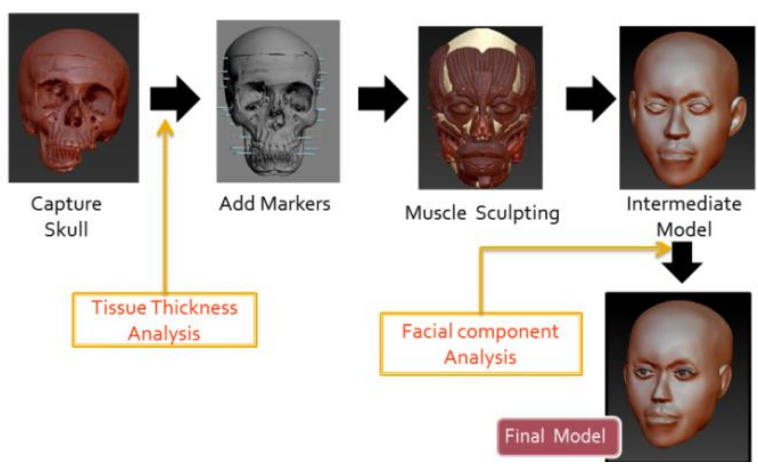

Figure 19: Summary of the process

Our solution is unique. Because It introduced facial reconstruction to Sri Lanka and it was based on Sri Lankan data (Tissue Thicknesses and Facial Features) based solution. It is a novel "Semi auto-mated" facial muscle sculpting based reconstruction method. We optimized the marker placements to 31 points where as Vanezis $\mathrm{P}$ (2000) used 40 [2] and Abate A (2004) used 19 points [5]. The inclusion of muscle reconstruction reduced the number of tissue thickness marker points in our method.

The quality of the solution can be assessed in the following way. It is a user friendly technique. Instead of sculpting, easy digital method is used. Expertise is now available in Sri Lanka and comprehensive manuals have been created for these 3D modelling software. It is scalable. If tissue thicknesses of other age groups are also available, it is easy to fit into any age category.

We used accepted standards and o ptimized the accepted tissue thickness landmarks to 31. Accepted face evaluation methods such as Facepool Comparison, Resemblance Rating, Photogrammetry Analysis, and Superimposition were used. Accepted Facial Muscle Anatomy Model was used. Standard Facial Component Comparison techniques 
such as Broca Agreement for Nasal Index and Halder Method for Eye Index were used.

\section{Conclusion}

Facial approximations for the female 20-30 year age group and medium weight cases were produced by this research. The outputs were successful and thoroughly evaluated using accepted evaluation techniques.

For the process, optimized tissue thickness landmarks and an accepted facial muscle anatomy based model were used. Although the research was performed on certain age and weight groups this process would be scalable to any other age/weight groups as well (given that the tissue thickness data for all those groups are collected).

Since all the attempted evaluation methods resulted in satisfactory outcomes it was concluded that the method adopted in this study to conduct the reconstruction is suitable and would ensure an accurate output. Hence the adopted procedure was identified to be recommendable to be adopted for future facial reconstructions in Sri Lanka.

Thus it was concluded that implementing a "National unit for facial reconstruction", which would adopt this procedure would be a very timely act for the island to uphold the rights of the deceased and their relatives.

Further, possible future incorporations are National Missing persons' database, Face recognition techniques, Facial Feature Component of CID.

Finally, this would be a solution for human identification for judicial medical officers, police investigators, archaeologists and also a relief for the relatives and friends of the missing person as facilitate the bereavement process .

\section{Acknowledgement}

Part of the findings has been published in the Galle Medical Journal. We acknowledge the services of the medical officers at the Radiology units, Central Hospital, Colombo, Durdans Group of Hospitals, and Teaching Hospital Colombo South and Prof R. Fernando Chair, Department of Forensic Medicine and Toxicology, Medical faculty, Colombo for their kind assistance.

\section{References}

1. Konica Minolta Optics, Inc. Konica minolta Non-contact 3D digitizer: Measuring instruments. http://www. konicaminolta.com/instruments/products /3d/non-contact/vivid910/optional_ accessories.html (5th July 2012)

2. Vanezis $P$, Vanezis M, Mccombe $G$, Niblett T. Facial reconstruction using 3-D computer graphics. Forensic Science International 2000; 108:81-95.

3. De Greef S, Claes P, Vandermeulen D, et al. Large-scale in-vivo Caucasian facial soft tissue thickness database for craniofacial reconstruction. Forensic Science International 2006; 159:126-146.

4. Rhine JS, Campbell HR. Thickness of facial tissues in American blacks. Journal of Forensic Sciences 1980;25: 847-58.

5. Abate A. F, Nappi M, Ricciardi S, Tortora G., Faces: 3D facial reconstruction from ancient skulls using content based image 
retrieval. Journal of Visual Languages \& Computing 2004;15(5):373-8.

\section{Contribution of authors}

Design to the study - MV, RNR, AKM, PIUA, VHP, ATD, and KDS

Supervision to the study-

MV, RNR, AKM, PIUA, VHP, ATD, KDS
Analysis of the data-

MV, RNR, AKM, PIUA, VHP, ATD, and KDS Interpretation of the results-

MV, RNR, AKM, PIUA, VHP, ATD, and KDS Writing the manuscript -

MV, RNR, AKM, PIUA, VHP, ATD, and KDS Revising the manuscript-

MV, RNR, AKM, PIUA, VHP, ATD, and KDS 